\title{
Quantum Phase Transitions in the Kitaev-Heisenberg Model on a Single Hexagon
}

\author{
D. GOTFRYD ${ }^{a, *}$ AND A.M. OLES $\hat{S}^{a, b}$ \\ ${ }^{a}$ M. Smoluchowski Institute of Physics, Jagiellonian University, prof. S. Łojasiewicza 11, PL-30348 Kraków, Poland \\ ${ }^{b}$ Max-Planck-Institut für Festkörperforschung, Heisenbergstr. 1, D-70569 Stuttgart, Germany
}

\begin{abstract}
We present a detailed analysis of the Kitaev-Heisenberg model on a single hexagon. The energy spectra and spin-spin correlations obtained using exact diagonalisation indicate quantum phase transitions between antiferromagnetic and anisotropic spin correlations when the Kitaev interactions increase. In cluster mean-field approach frustrated nearest neighbor exchange stabilizes the stripe phase in between the Néel phase and frustrated one which evolves towards the Kitaev spin liquid.
\end{abstract}

DOI: 10.12693/APhysPolA.127.318

PACS: 75.10.Jm, 75.25.Dk, 75.30.Et

\section{Introduction}

Possible realizations of quantum spin liquids is one of the most intriguing questions in modern theory of frustrated spin systems [1-3]. One of the prominent examples of spin liquid was introduced by Kitaev [4]. As a unique feature of this exactly solvable model spin-spin correlations are finite only on nearest neighbor (NN) bonds [5]. Recently a lot of attention is devoted to frustrated spin models on the honeycomb lattice, either to $J_{1}-J_{2}$ Heisenberg interactions [6, 7], or to Kitaev-Heisenberg $(\mathrm{KH})$ model [8-12]. The latter is motivated by $\mathrm{A}_{2} \mathrm{IrO}_{3}$ iridates $(\mathrm{A}=\mathrm{Na}, \mathrm{Li})$ which is a candidate to host Kitaev model physics. For a realistic description of these compounds, and in particular of the observed zigzag phase [13], also next nearest neighbor (NNN) and third nearest neighbor $(3 \mathrm{NN})$ Heisenberg antiferromagnetic (AF) interactions frustrating the Néel state are necessary $[6,10]-$ these terms are also justified by rather itinerant character of the electrons in $\mathrm{A}_{2} \mathrm{IrO}_{3}$ [14]. Several experiments suggest that the NNN $\left(J_{2}\right)$ and $3 \mathrm{NN}\left(J_{3}\right)$ coupling constants have similar values, i.e., $J_{2} \approx J_{1} / 2, J_{3} \approx J_{2}$ [10].

The purpose of this paper is to investigate the evolution of spin-spin correlations on a single hexagon when interactions change from AF Heisenberg to highly frustrated ferromagnetic (FM) Kitaev ones. This evolution is modified when a cluster mean-field (MF) approach is applied, similar to the one used before for the $J_{1^{-}} J_{2^{-}} J_{3}$ model [6] and the Kugel-Khomskii model [15].

\section{Model}

The KH Hamiltonian has the form [10]:

$$
\begin{aligned}
H & \equiv-2 J \alpha \sum_{\langle i j\rangle \| \gamma} S_{i}^{\gamma} S_{j}^{\gamma}+J(1-\alpha)\left\{\sum_{\langle i j\rangle} \boldsymbol{S}_{i} \cdot \boldsymbol{S}_{j}\right. \\
& \left.+J_{2} \sum_{\langle\langle i j\rangle\rangle} \boldsymbol{S}_{i} \cdot \boldsymbol{S}_{j}+J_{3} \sum_{\langle\langle\langle i j\rangle\rangle\rangle} \boldsymbol{S}_{i} \cdot \boldsymbol{S}_{j}\right\} .
\end{aligned}
$$

\footnotetext{
${ }^{*}$ corresponding author; e-mail: dorota.gotfryd@uj.edu.pl
}

In the first Kitaev term, bond-dependent Ising-like interactions are selected by $\gamma \in\{x, y, z\}$ depending on the bond direction. The parameter $\alpha$ interpolates between the Heisenberg $(\alpha=0)$ and the Kitaev $(\alpha=1)$ interactions. We set the energy unit $J=1$, and we take equal NNN $\left(J_{2}\right)$ and $3 \mathrm{NN}\left(J_{3}\right)$ interactions, i.e., $J_{2}=J_{3}=J_{1} / 2$ and $J_{1} \equiv(1-\alpha) J$. Following the $a b$ initio calculations [14], we select the AF NN Heisenberg terms and FM Kitaev ones. Note that already at small $\alpha>0$ spin interactions are anisotropic, and classically Néel or resonating valence bond (RVB) phase is destroyed at $\alpha=1 / 3$ when some NN interactions switch from AF to FM. Here we investigate the more challenging quantum case.

We performed exact diagonalisation (at $T=0$ ) and investigated the energy spectra and spin correlations between NN, NNN, and 3NN spins at sites $\{i, j\}$ :

$$
S(i, j)=\left\langle\boldsymbol{S}_{i} \cdot \boldsymbol{S}_{j}\right\rangle=\frac{1}{d} \sum_{k=1}^{d}\left\langle\Phi_{k}\left|\boldsymbol{S}_{i} \cdot \boldsymbol{S}_{j}\right| \Phi_{k}\right\rangle,
$$

where $\left\{\left|\Phi_{k}\right\rangle\right\}$ are individual degenerate states in the ground state manifold, and $k=1, \ldots, d$. In addition, we investigate below partial spin correlations which reflect the anisotropic character of spin interactions,

$$
S^{\gamma}(i, j)=\left\langle S_{i}^{\gamma} S_{j}^{\gamma}\right\rangle=\frac{1}{d} \sum_{k=1}^{d}\left\langle\Phi_{k}\left|S_{i}^{\gamma} S_{j}^{\gamma}\right| \Phi_{k}\right\rangle .
$$

For a free hexagon, no order may occur and $\left\langle S_{i}^{z}\right\rangle \equiv 0$.

In the quest of quantum phase transitions (QPTs) several trails have been revealed. First clue appears to be change of the ground state of the Hamiltonian operator which defines the QPT. Second track signalling directly the transition is the variation of spin-spin correlations either the change of sign, or discontinuities which are fingerprints of QPTs. Finally, extremal values of the ground state energy $E_{0}$ might also indicate a transition [9].

\section{Results and discussion}

Spin-spin correlations change in a discontinuous way at some values of $\alpha$ which indicate QPTs. Here we 
show only the correlations for $\mathrm{NN}$ and for $3 \mathrm{NN}$ which are sufficient to conclude about the QPTs when $\alpha$ increases, see Fig. 1. First, for $\alpha \in[0,0.355)$ (phase I),

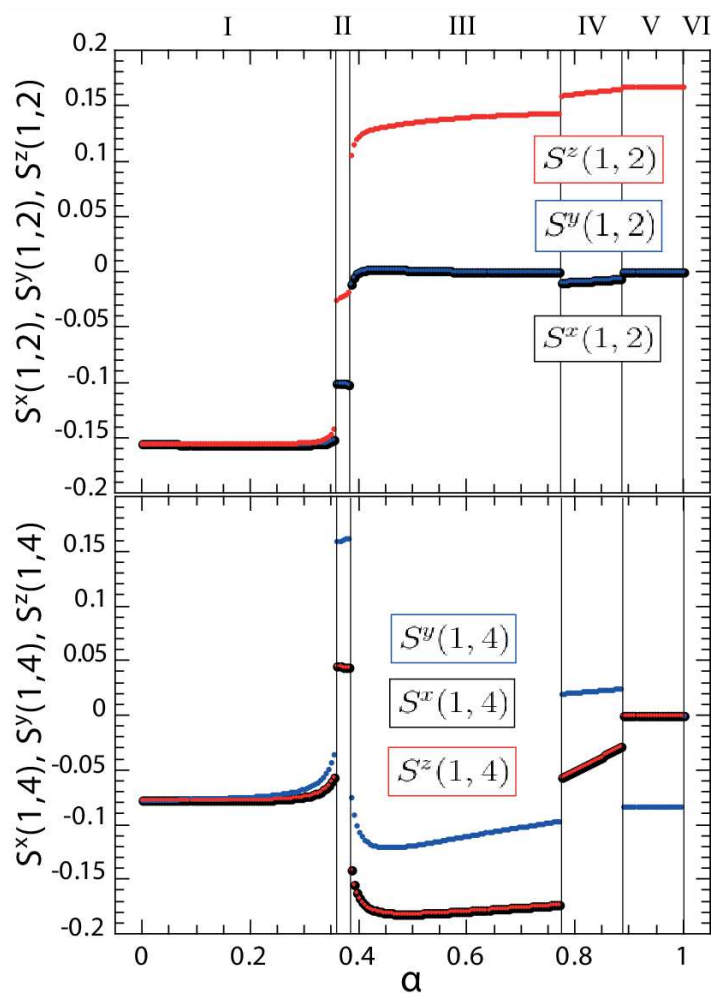

Fig. 1. Spin-spin correlations as obtained for $\mathrm{KH}$ model (1) at increasing $\alpha$, with $-2 J S_{1}^{z} S_{2}^{z}$ interaction at $\alpha=1$ : top (a) - NN $\left\langle S_{1}^{\gamma} S_{2}^{\gamma}\right\rangle$, and bottom (b) $3 \mathrm{NN}\left\langle S_{1}^{\gamma} S_{4}^{\gamma}\right\rangle$ (this bond is parallel to $\left\langle S_{2}^{y} S_{3}^{y}\right\rangle$ at $\alpha=1$ ). QPTs occur at vertical lines and phases are labeled from I to VI.

the NN correlations are almost independent of $\gamma$, i.e., $S^{\gamma}(1,2) \simeq S(1,2) / 3$, and one finds a RVB phase which weakens above $\alpha \simeq 0.3$. At $\alpha \simeq 0.355$ the first $\mathrm{QPT}$ occurs, see Fig. 1a and b, and both $S(1,3)$ and $S(1,4)$ change signs, cf. Fig. 2a and b. Two nondegenerate states cross at the QPT and the derivative of $E_{0}$ changes (Table). As in spin-orbital systems [15], phase II is driven here by $J_{2}$ and $J_{3}$ while $J_{1}$ changes sign. It has FM (AF) NNN (3NN) correlations, see Fig. 2b, and we suggest that it is a precursor of the zigzag phase found in this range of parameters $[10,13]$.

A second QPT occurs at $\alpha \simeq 0.385$, where two nondegenerate ground states intersect and $E_{0}$ is maximal. Here both spin-spin correlations $S(1,2)$ and $S(1,4)$ change signs. Already at $\alpha=0.355$ we observe that $S^{z}(1,2)$ separates from $S^{x}(1,2)=S^{y}(1,2)$, and $S^{y}(1,4)$ separates from $S^{x}(1,4)=S^{z}(1,4)$, and this persists up to $\alpha=1$, see Fig. $1 \mathrm{a}$ and $\mathrm{b}$.

Further discontinuities arise for all $S^{\gamma}(1,2)$ at $\alpha \simeq$ 0.770 , but in their sum $S(1,2)$ they nearly cancel one another and the discontinuity of $S(1,2)$ almost vanishes. At this QPT a singlet and a triplet cross. Notably, the correlation functions do not change signs at this QPT,

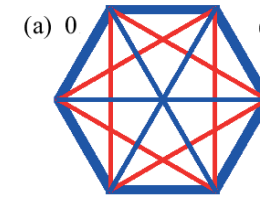

(d) 0.77

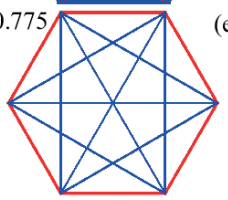

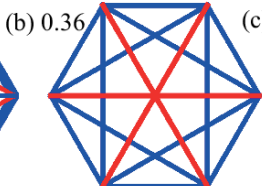

(e)

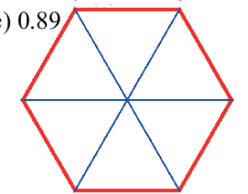

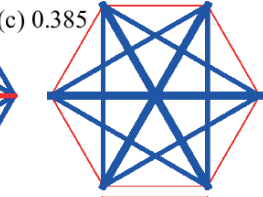

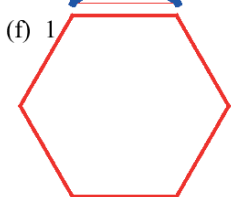

Fig. 2. Spin-spin correlation functions for the Hamiltonian (1) for selected values of $\alpha$ (below each part). The sign (AF or FM) is indicated by line color: blue - AF correlation, red - FM correlation; the line width is proportional to its absolute value. Parameters: $J_{2}=J_{3}=0.5 J_{1}$.

see Fig. 2c and d. We observe that in phase IV NN FM correlations grow stronger while NNN and 3NN correlations (both $\mathrm{AF}$ ) weaken.

At $\alpha \simeq 0.890$ the triplet state crosses with another singlet ground state, indicating a QPT to a distinct spin disordered phase $\mathrm{V}$, stable for $\alpha \in[0.89,1)$. All spin-spin correlations are discontinuous at the transition (Table) and all NNN ones vanish, see Fig. $2 \mathrm{e}$, while $S^{y}(1,4)$ is small and finite [16], see Fig. 1b. The gap between the ground state and triplet excited state first grows and then starts to shrink with increasing $\alpha$ until both states merge at $\alpha=1$, where one finds FM spin correlations for NN only, see Fig. 2f. The only finite spin-spin correlation at $\alpha=1$ happens to be $S^{z}(1,2)$, see Fig. 1 a.

\section{TABLE}

Discontinuities in spin-spin correlations $S(1, n)$ and the feature of the ground state energy $E_{0}$ (if any) at five QPTs which occur at $\alpha_{\mathrm{c}}$. At the first three QPTs spin correlations change sign (sign) between the ground states with degeneracies $d_{<}$and $d_{>}$for $\alpha<\alpha_{\mathrm{c}}$ and $\alpha>\alpha_{\mathrm{c}}$, respectively.

\begin{tabular}{c|c|c|c|c|c}
\hline \hline$\alpha_{\mathrm{c}}$ & $S(1, n)$ & Sign & $d_{<}$ & $d_{>}$ & Feature of $E_{0}$ \\
\hline$\sim 0.355$ & $S(1,3)$ & $+/-$ & 1 & 1 & slope change \\
& $S(1,4)$ & $-/+$ & & & \\
$\sim 0.385$ & $S(1,2)$ & $-/+$ & 1 & 1 & maximum \\
& $S(1,4)$ & $+/-$ & & & \\
$\sim 0.770$ & $S(1,4)$ & $+/-$ & 1 & 3 & slope change \\
$\sim 0.890$ & $S(1, n)$ & $\ldots$ & 3 & 1 & $\ldots$ \\
1.0 & $S(1,4)$ & $-/ 0$ & 1 & 4 & $\ldots$
\end{tabular}

For $\alpha=1$ the ground state degeneracy is $d=4$; it is lifted when minute Heisenberg interaction is added at $\alpha<1$, in analogy to the 2D compass model, where Heisenberg terms remove high degeneracy of the ground state [17]. In contrast, however, the ground state does not change and the Kitaev spin liquid survives here in the range of $\alpha \in[0.89,1)$, with additional 3NN correlations.

Special attention has to be paid to $S^{y}(1,4)$, with its sign being different from that of $S^{x}(1,4)=S^{z}(1,4)$ when $\alpha \in[0.355,1)$. This function has a discontinuity 
at each QPT, see Table. It concerns the bond $\langle 14\rangle$ which is parallel to the NN bond $\langle 23\rangle$ with $S_{2}^{y} S_{3}^{y}$ interaction in the Kitaev limit, so we see that the Kitaev part induces $3 \mathrm{NN}$ correlations for the same component $\gamma$ which is active along the NN bonds parallel to it. Partial NNN spin correlations also separate at $\alpha=0.355$ but drop to zero when spins get disordered at $\alpha=0.890$.

Previous studies within the cluster MF $[6,15]$ inspired us to consider the hexagon with only NN Heisenberg $J_{1}$ and Kitaev $J_{K} \equiv-2 J \alpha$ terms. We embedded the hexagon by the MF terms, replacing spins along outer NN bonds with the order parameters, $s_{i}^{z} \equiv\left\langle S_{i}^{z}\right\rangle$. They were selected using either Néel or stripe ansatz and calculated self-consistently. For $\alpha \in(0,0.39]$ (phase II) the $\mathrm{SU}(2)$ symmetry is broken and $\left\{s_{i}^{z}\right\}$ and $\{S(i, j)\}$ follow Néel AF order (phase I) which extends up to $\alpha=0.395$ due to quantum fluctuations, see Fig. 3. Near the QPT at $\alpha=0.390$ one finds robust Néel order with positive/negative values of $\left|s_{i}^{z}\right| \simeq 0.4172$ at odd/even site $i$ of the hexagon.

For $\alpha \leq 0.36$ the stripy ansatz gave $s_{i}^{z}=0$, while spinspin correlations are constant and RVB-like. At $\alpha=$ 0.365 the symmetry is broken $\left(s_{i}^{z} \neq 0\right)$, but the NN correlations do not follow the stripy pattern yet. We obtained the stripy phase for $\alpha \in[0.395,0.55]$ (phase III), with FM (AF) spin-spin correlations $S(1,2)=S(4,5)$ (otherwise), see Fig. 3. Unlike in Néel phase, here one finds two distinct values of the order parameters $\left\{\left|s_{i}^{z}\right|\right\}$, e.g. $s_{i}^{z} \simeq 0.3795(-0.2675)$ for $i=1,2,4,5(i=3,6)$ at $\alpha=0.395$, as the sites are nonequivalent and the latter ones are exposed to enhanced quantum fluctuations within the hexagon. These fluctuations disappear at $\alpha=0.5$, in agreement with the mapping on the FM Heisenberg model [9]. Unfortunately, we could not obtain converged results for $\alpha \in[0.5,0.525)$. The region of (stripe) phase III agrees partly with that obtained for a larger cluster of $N=24$ sites, $\alpha \in[0.4,0.8][9]$. We thus conclude that the stripy order is subtle and hard to stabilize on a single hexagon.

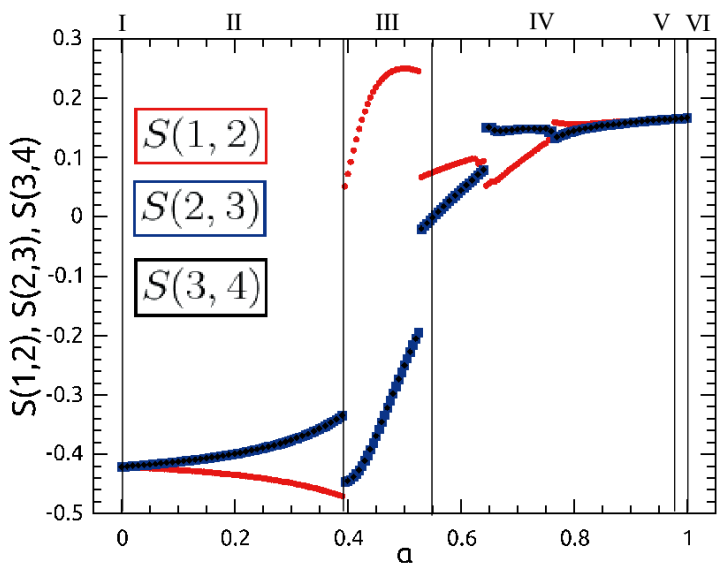

Fig. 3. Spin-spin NN correlations obtained for converged MF calculations for $0 \leq \alpha \leq 1$ : $S(1,2), S(2,3)$, $S(3,4)$. QPTs occur at vertical lines and phases are labeled from I to VI. Parameters: $J_{2}=J_{3}=0$.
For $\alpha \in(0.555,0.98)$ (phase IV) the symmetry remains broken but the stripe phase is destroyed here by the Kitaev terms and all NN $S(i, j)$ are weakly FM and anisotropic, see Fig. 3. At $\alpha=0.98$ one finds a QPT to disordered spin liquid with $d=3$ (phase V). It is similar to phase IV of a free hexagon (see Table). The last QPT is found at the Kitaev limit $\alpha=1$ itself, where we find again $d=4$.

\section{Summary}

We conclude that increasing Kitaev interactions cause spin-spin correlations $S_{i}^{\gamma} S_{j}^{\gamma}$ to separate. This phenomenon is generic and occurs both for a free hexagon and in MF shortly after one NN interaction $S_{i}^{\gamma} S_{j}^{\gamma}$ changes sign. Unless the Kitaev terms dominate, investigation of possible long-range order requires cluster MF or even more sophisticated methods. The Kitaev spin liquid phase extends to $\alpha<1$ also in the MF approach, but $3 \mathrm{NN}$ spin correlations are induced in this regime.

\section{Acknowledgments}

We thank Jiři Chaloupka for insightful discussions and acknowledge support by the Polish National Science Center (NCN) under Project No. 2012/04/A/ST3/00331.

\section{References}

[1] B. Normand, Cont. Phys. 50, 533 (2009).

[2] L. Balents, Nature (London) 464, 199 (2010).

[3] A.M. Oleś, J. Phys. Condens. Matter 24, 313201 (2012); Acta Phys. Pol. A 127, 163 (2015).

[4] A.Y. Kitaev, Ann. Phys. (New York) 321, 2 (2006).

[5] G. Baskaran, S. Mandal, R. Shankar, Phys. Rev. Lett. 98, 247201 (2007).

[6] A.F. Albuquerque, D. Schwandt, B. Hetényi, S. Capponi, M. Mambrini, A.M. Läuchli, Phys. Rev. B 84, 024406 (2011).

[7] R. Ganesh, J. van den Brink, S. Nishimoto, Phys. Rev. Lett. 110, 127203 (2013).

[8] G. Jackeli, G. Khaliullin, Phys. Rev. Lett. 102, 017205 (2009).

[9] J. Chaloupka, G. Jackeli, G. Khaliullin, Phys. Rev. Lett. 105, 027204 (2010); Phys. Rev. Lett. 110, 097204 (2013).

[10] I. Kimchi, Y. You, Phys. Rev. B 84, 180407 (2011).

[11] R. Schaffer, S. Bhattacharjee, Y.B. Kim, Phys. Rev. B 86, 224417 (2012).

[12] J.G. Rau, E.K.-H. Lee, H.-Y. Kee, Phys. Rev. Lett. 112, 077204 (2014).

[13] F. Trousselet, M. Berciu, A.M. Oleś, P. Horsch, Phys. Rev. Lett. 111, 037205 (2013).

[14] K. Foyevtsova, H.O. Jeschke, I.I. Mazin, D.I. Khomskii, R. Valentí, Phys. Rev. B 88, 035107 (2013).

[15] W. Brzezicki, J. Dziarmaga, A.M. Oleś, Phys. Rev. Lett. 109, 237201 (2012); Phys. Rev. B 87, 064407 (2013).

[16] F. Trousselet, G. Khaliullin, P. Horsch, Phys. Rev. B 84, 054409 (2011).

[17] F. Trousselet, A.M. Oleś, P. Horsch, Europhys. Lett. 91, 40005 (2010); Phys. Rev. B 86, 134412 (2012). 